\title{
Análise de desempenho de sistemas de produção modais de pecuária de cria no Brasil
}

\author{
João Carlos Correia Baptista Soares de Mello ${ }^{\mathrm{a} *}$, Eliane Gonçalves Gomes ${ }^{\mathrm{b}}$, \\ Urbano Gomes Pinto de Abreuc, Thiago Bernardino de Carvalho ${ }^{\mathrm{d}}$, Sérgio de Zen ${ }^{\mathrm{e}}$ \\ a*jcsmello@pesquisador.cnpq.br, UFF, Brasil \\ beliane.gomes@embrapa.br, Embrapa Sede, Brasil \\ curbano@cpap.embrapa.br, Embrapa Pantanal, Brasil \\ dtbcarval@cepea.org.br, USP, Brasil \\ 'sergdzen@usp.br, USP, Brasil
}

\section{Resumo}

Neste artigo propõe-se analisar comparativamente o desempenho de sistemas de produção modais de pecuária extensiva de corte na fase de cria em 21 municípios brasileiros. A cria é a fase da atividade que sustenta toda a cadeia de produção da pecuária de corte. É usado o modelo de análise envoltória de dados (DEA) com retornos variáveis de escala. 0 objetivo é medir o desempenho da decisão do produtor em relação à composição do sistema produtivo. As variáveis usadas são número de reprodutores, quantidade de bezerros e vacas de descarte. Dos 21 sistemas avaliados, quatro foram DEA BCC eficientes e a maioria dos sistemas avaliados opera na região de retornos crescentes de escala. Palavras-chave

Eficiência. DEA. Pecuária. Sistemas modais.

\section{Introdução}

Segundo Euclides Filho (2000), sistema de produção de gado de corte é o conjunto de tecnologias e práticas de manejo, tipo de animal, propósito da criação, raça ou grupamento racial e ecorregião onde a atividade é desenvolvida. Na definição de um sistema de produção devem-se considerar os aspectos sociais, econômicos e culturais, pois possuem influência decisiva nas modificações que poderão ser impostas por forças externas. Também influenciam a forma como as mudanças para tornar o processo eficiente devem ser implementadas e, assim, como as transformações alcançam os benefícios esperados. Em paralelo, devem-se definir o mercado e a demanda a ser atendida (quais são e como devem ser atendidos os consumidores).

A estrutura central na cadeia produtiva da pecuária de corte é o sistema biológico de produção de bovinos, englobando as diferentes etapas da criação (cria, recria e engorda), em combinações em torno das quais se agrupam os produtores (CARDOSO, 1994).
No Brasil, a fase de cria da pecuária de corte ocorre em regime predominantemente extensivo, com a base da alimentação sendo pastagens nativas e/ou cultivadas, englobando: bezerros(as) até a desmama, ou até um ano de idade; vacas; novilhas (em recria ou com idade de cobertura); touros.

Considerando-se isoladamente as fases da pecuária de corte, em sistemas de produção considerados representativos da média, após análises de benefício/custo pôde-se concluir que a cria constitui-se na atividade de menor rentabilidade, além de ser aquela que apresenta o maior risco. Todavia, é também a que sustenta toda a estrutura da cadeia de produção da pecuária de corte (EUCLIDES FILHO, 1996).

Apesar de a importância da visão sistêmica na agropecuária ser incontestável, ainda não está estabelecida na pesquisa agropecuária brasileira. 0 presente estudo, por meio de modelagem utilizando análise envoltória de dados (DEA), busca avaliar comparativamente o desempenho dos sistemas de 
produção modais de pecuária extensiva de corte na sua fase de cria em alguns municípios do Brasil. 0 objetivo é medir o desempenho da decisão do produtor em relação à composição do sistema produtivo, que tem impacto direto nos custos e na renda do sistema.

\section{Estudo de caso}

Os dados primários foram levantados por meio do sistema de painel, que permite a definição de propriedades representativas, conforme Plaxico e Tweeten (1963). Para estudos de unidades produtivas do meio rural, esses mesmos autores descrevem o sistema de fazendas representativas como o ideal. Porém algumas definições e suposições devem ser adotadas, as características devem ser constantemente revistas e os dados de produção frequentemente revisados para refletir os avanços tecnológicos.

Apesar da dificuldade de caracterizar uma única propriedade e sistema de produção que sejam representativos da localidade em estudo (aqui município), o método busca, por meio da experiência dos produtores participantes, caracterizar a propriedade mais comumente encontrada na região (CARVALHO; ZEN; FERREIRA, 2008). Em algumas áreas, a impossibilidade de determinar essa tipicidade fez com que mais de uma propriedade/sistema de produção representado fosse estabelecido. A execução do painel segue quatro etapas principais:

- Etapa 1: Levantamento de coeficientes técnicos de produção e de informações regionais;

- Etapa 2: Visita às propriedades;

- Etapa 3: Preparo de planilhas eletrônicas; e

- Etapa 4: Realização do painel.

As informações obtidas nas etapas 1 e 2, referentes aos processos operacionais que determinam os custos de produção e a produtividade, proporcionam a elaboração de planilhas eletrônicas (previamente preenchidas) destinadas a facilitar o funcionamento da etapa 4.

0 painel é um procedimento de obtenção de informações menos oneroso que o levantamento censitário ou amostral de unidades agrícolas. Outra vantagem é que proporciona maior agilidade e versatilidade na atualização dos dados sem comprometer a sua qualidade. A técnica consiste em uma reunião com um grupo formado por um ou mais pesquisadores, um técnico regional e oito pecuaristas (em média), e pode variar de cinco a dez produtores. As reuniões foram marcadas com antecedência, utilizando-se geralmente como contato os sindicatos rurais regionais. Os temas e os números, determinados previamente em entrevistas com os técnicos locais, foram debatidos com os produtores rurais.

Para que todos os participantes interagissem, utilizou-se um computador portátil e um aparelho destinado a projetar a planilha previamente elaborada (etapa 3). Diferentes coeficientes técnicos (quantidade de insumos), preços e frequências de uso foram apresentados aos grupos, que discutiram e aperfeiçoaram as informações. Ao final desse debate, pode-se dizer que todas as caracterizações de propriedades típicas regionais tiveram o aval dos produtores rurais. Com isso, os índices de produtividade, custos de implantação, custos fixos e variáveis, ou seja, todos os números resultantes do painel tendem a ser bastante próximos da realidade regional.

Destaca-se: índices e custos declarados por cada participante não estão relacionados com a propriedade de cada um mas com uma única, declarada no início do painel como a que representa melhor o tamanho e o sistema de produção da maioria das propriedades locais (CARVALH0; ZEN; TAVARES, 2009).

Neste trabalho foram avaliados 21 sistemas modais de produção de gado de corte que realizavam apenas a fase de cria em sete estados do Brasil. Os dados, oriundos do projeto Indicadores Pecuários desenvolvido pelo Centro de Estudos Avançados em Economia Aplicada (CEPEA) e pela Confederação da Agricultura e Pecuária do Brasil (CNA), foram coletados em municípios destes sete estados: Mato Grosso do Sul (oito); Goiás (quatro); Rio Grande do Sul (um); Minas Gerais (quatro); Tocantins (dois); São Paulo (um); e Bahia (um). Para a coleta dos dados foram realizados painéis com produtores e com a assistência técnica local, segundo a metodologia descrita em documento do Centro de Estudos Avançados em Economia Aplicada (CENTRO..., 2010).

Uma análise preliminar dos resultados desses painéis com uso de técnicas multivariadas e com o objetivo de classificar os diferentes sistemas modais pode ser vista em Abreu, Carvalho e Zen (2010).

\section{Análise de envoltória de dados - DEA}

\subsection{Aspectos gerais}

DEA é tradicionalmente uma metodologia de análise de eficiência que usa programação linear. Seu objetivo é calcular a eficiência de unidades produtivas, chamadas de unidades de tomada de decisão ou DMUs (Decision Making Units), conhecendo-se os níveis de recursos empregados e de resultados obtidos. 
DEA otimiza cada observação individual de modo a estimar uma fronteira eficiente (linear por partes), composta das unidades que apresentam as melhores práticas dentro da amostra em avaliação (unidades Pareto-Koopmans eficientes). Essas unidades servem como referência ou benchmark para as unidades ineficientes.

Os dois modelos mais conhecidos de DEA são o CCR (CHARNES; COOPER; RHODES, 1978) e o BCC (BANKER; CHARNES; COOPER, 1984). 0 modelo CCR assume a hipótese de retornos constantes à escala. 0 modelo BCC considera retornos variáveis à escala, ou seja, substitui o axioma da proporcionalidade pelo axioma da convexidade. Tradicionalmente são possiveis duas orientações radiais para esses modelos na busca da fronteira de eficiência: orientação a inputs (busca-se minimizar os recursos mantendo-se inalterados os níveis de produção) e orientação a outputs (implica aumentar os produtos sem alterar a quantidade de insumos usados).

Os modelos (1) e (2) representam a forma linearizada dos modelos DEA BCC dos Multiplicadores (a) e do Envelope (b), orientados a inputs e a outputs, respectivamente. Nas formulações (1a) e (2a), Eff é a eficiência da DMU o em análise; $x_{i k}$ representa o input i da DMU k, $\mathrm{y}_{\mathrm{jk}}$ representa o output j da DMU k; $v_{i}$ é o peso atribuído ao input $i$; $u_{j}$ é o peso atribuído ao output $\mathrm{j} ; \mathrm{x}_{\mathrm{io}}$ e $\mathrm{y}_{\mathrm{jo}}$ são input i e output $\mathrm{j}$ da DMU $o$, respectivamente; $u^{*}$ e $v^{*}$ são os fatores de escala (quando positivos indicam retornos decrescentes de escala; quando negativos indicam retornos crescentes de escala; quando nulos indicam retornos constantes de escala). Em (1b) e (2b), $h_{0}$ é a eficiência da DMU o em análise (note-se que os PPLs (a) e (b) são duais e, dessa forma, têm o mesmo valor da função objetivo); $\lambda_{\kappa}$ é a contribuição da DMU k na formação do alvo da DMU o (as DMUs com $\lambda_{\kappa}$ não nulo são os benchmarks da DMU o).

Os modelos CCR, orientados a inputs e a outputs, em suas formulações dual e primal, podem ser obtidos dos modelos (1) e (2) omitindo-se a restrição de convexidade no modelo do Envelope $\left(\sum_{k} \ddot{e}_{k}=1\right)$ e os fatores de escala no modelo dos Multiplicadores.

Min $\quad \operatorname{Eff}_{o}=\sum_{j} u_{j} y_{j o}-u^{*}$

sujeito a

$\sum_{i} v_{i} x_{i o}=1$

$-\sum_{i} v_{i} x_{i o}+\sum_{j} u_{j} y_{j o}-u^{*} \leq 0, \forall k$

$u, v \geq 0, u^{*} \in \Re$
$\operatorname{Min} h_{o}$

sujeito a

$h_{o} x_{i o}-\sum_{k} x_{i k} \lambda_{k} \geq 0, \forall i$

$-y_{j o}+\sum_{k} y_{j k} \lambda_{k} \geq 0, \forall j$

$\sum_{k} \lambda_{k}=1$

$\lambda_{k} \geq 0, \forall k$

Min $E f f_{o}=\sum_{i} v_{i} x_{i o}-v^{*}$

sujeito a

$\sum_{j} u_{j} y_{j o}=1$

$-\sum_{i} v_{i} x_{i o}+\sum_{j} u_{j} y_{j o}+v^{*} \leq 0, \forall k$

$u, v \geq 0, v^{*} \in \Re$

$\operatorname{Max} h_{o}$

sujeito a

$x_{i o}-\sum_{k} x_{i k} \lambda_{k} \geq 0, \forall i$

$-h_{o} y_{j o}+\sum_{k} y_{j k} \lambda_{k} \geq 0, \forall j$

$\sum_{k} \lambda_{k}=1$

$\lambda_{k} \geq 0, \forall k$

Para modelar e interpretar corretamente os resultados de DEA é necessário conhecer as propriedades de seus modelos. Duas das mais importantes são (GOMES; SOARES DE MELLO; MANGABEIRA, 2009; GOMES et al. 2009b):

- Em qualquer modelo DEA, a DMU que apresentar a melhor relação (output j)/(input i) será sempre eficiente. Essa propriedade obriga a que na formulação do modelo DEA seja necessário verificar a existência de relação causal entre cada output e cada input. A não observância dessa relação pode conduzir a resultados sem sentido; $\mathrm{e}$

- 0 modelo CCR, que na sua forma fracionária é uma função homogênea de grau zero, tem como propriedade principal a proporcionalidade entre inputs e outputs na fronteira. Isto tem como consequência que o aumento (decremento) na quantidade dos inputs provocará acréscimo (redução) proporcional no valor dos outputs.

\subsection{Modelos DEA com restrições aos pesos}

Os modelos DEA clássicos permitem total liberdade na escolha dos pesos que darão o máximo valor de eficiência a uma dada DMU. Essa liberdade é 
importante na identificação das unidades ineficientes, ou seja, daquelas DMUs que apresentam baixo desempenho inclusive com seu próprio conjunto de multiplicadores.

A flexibilidade na escolha dos pesos é uma das vantagens atribuídas à modelagem DEA. Entretanto, os pesos calculados podem ser inconsistentes com o conhecimento em relação aos valores relativos de inputs e outputs. Assim, a incorporação de julgamentos de valor dos especialistas a respeito da importância relativa de cada variável no cálculo das medidas de eficiência surge como uma evolução natural das aplicações de DEA a problemas reais. Ou seja, há a necessidade de introduzir condições além das de não negatividade.

Quando há preferências entre os inputs e/ou outputs por parte dos agentes de decisão, esses julgamentos de valor são incorporados aos modelos DEA por meio de restrições aos pesos (ou multiplicadores) associados aos inputs e/ou aos outputs das unidades avaliadas. Allen et al. (1997) e Thanassoulis, Portela e Allen (2004) apresentam uma revisão da evolução da incorporação de julgamentos de valor através de restrições aos pesos. Angulo Meza e Lins (2002) consideram que a adição de restrições aos pesos é uma das técnicas que promovem melhoria de discriminação em DEA, com a incorporação de opiniões subjetivas dos decisores. Adler, Friedman e Sinuany-Stern (2002) também incluem as restrições aos pesos dentro das técnicas de melhora de discriminação em DEA e apresentam vários tipos de restrições. As principais são (LINS; ANGULO MEZA, 2000): (a) restrições diretas sobre os multiplicadores; (b) ajuste dos níveis de input-output observados para a captura de julgamentos de valor; (c) restrição a inputs e outputs virtuais.

Um dos tipos de modelo de restrições aos pesos é o modelo de Regiões de Segurança Tipo 1 (Assurance Region Type 1 - ARI), conforme definido em Thompson et al. (1990). Em (3) apresenta-se a formulação genérica do modelo BCC com as restrições ARI impostas aos outputs, onde $\alpha_{\mathrm{i}}$ e $\beta_{\mathrm{i}}$ são constantes especificadas pelo decisor e que, após uma conveniente normalização, refletem os julgamentos de valor sobre a importância relativa entre os outputs i e i + 1. Este é o modelo usado neste artigo.

$$
\begin{aligned}
& \operatorname{Max} \quad \sum_{\mathrm{j}} u_{j} y_{j o}-u^{*} \\
& \text { sujeito a } \\
& \sum_{i} v_{i} x_{i o}=1 \\
& -\sum_{i} v_{i} x_{i k}+\sum_{j} u_{j} y_{j k}-u^{*} \leq 0, \forall k \\
& \alpha_{i} \leq \frac{v_{i}}{v_{i+1}} \leq \beta_{i} \\
& u_{j}, \quad v_{i} \geq 0, \forall j, i \\
& u^{*} \in \Re
\end{aligned}
$$

\subsection{Artigos correlatos: DEA em avaliação de pecuária no Brasil}

É recente a utilização de modelos DEA com objetivo de modelar a eficiência dos sistemas de produção de gado de corte no Brasil (GOMES, 2008). Abreu et al. (2006) analisaram a eficiência de um sistema de produção de gado de corte no Pantanal de Corumbá no qual foram implantadas tecnologias ao longo de oito anos (1995-2002). Foram consideradas informações sobre dez categorias de gastos com insumos (inputs) e uma categoria de produto (output). Os autores usaram a técnica multivariada de componentes principais com o objetivo de diminuir o número das variáveis de input. A metodologia identificou as fontes das ineficiências em função dos gastos desnecessários com insumos, aspecto fundamental para o produtor na tomada de decisões. Adicionalmente, possibilitou a análise dos anos eficientes em relação aos ineficientes, de modo que os eficientes serviram como referência para adoção de novas práticas de manejo zootécnico e de equilíbrio nos gastos com insumos.

Com os mesmos dados econômicos registrados no período citado acima, Abreu et al. (2008) avaliaram duas metodologias de escolha das variáveis para a realização da análise DEA: (a) uma variante do método multicritério de seleção de variáveis que combina os objetivos conflitantes de melhor ordenação das unidades de decisão e maior eficiência média; (b) técnicas de estatística multivariada. Nos dois modelos resultantes (com seis inputs e um output; e com três inputs e um output, respectivamente) foram analisados os resultados de eficiência em relação às fronteiras DEA clássica e invertida, além do valor do índice composto que agrega ambos os resultados. 0 melhor modelo, avaliado pela análise de tendência linear, foi obtido com a variante proposta do método multicritério de seleção de variáveis cujo índice composto resultou em estimativas significativas dos coeficientes de regressão $(0,046)$ e de determinação $(0,700)$.

Técnicas semelhantes de seleção de variáveis em DEA foram usadas por Abreu et al. (2012). Nesse caso, o objetivo foi avaliar quais as unidades que fizeram melhor uso de uma linha de crédito que visava promover a retenção de matrizes nos rebanhos pantaneiros.

Os modelos DEA de input unitário, descritos por Caporaletti, Dulá e Womer (1999), Soares de Mello et al. (2008a) e Soares de Mello, Angulo Meza e Branco da Silva (2009) foram usados por Gomes et al. (2012) para avaliar pecuaristas do Pantanal usando variáveis zootécnicas e produtivas. Para evitar avaliação concentrada em um único indicador por unidade, o estudo usou também a fronteira invertida, com a modelagem descrita por Soares de Mello et al. (2008b). 
Deve-se ressaltar que há também estudos de eficiência na pecuária brasileira sem o uso de DEA. Por exemplo, Lampert et al. (2012) usaram a rentabilidade financeira para avaliação da pecuária de corte no Rio Grande do Sul.

\section{Modelagem}

Na estruturação de um modelo DEA é necessário definir as unidades a avaliar (DMUs), as variáveis que constituirão o modelo (inputs e outputs) e o modelo DEA adotado (CCR, BCC, entre outros; orientação a inputs, a outputs etc.).

\subsection{DMUs}

0 objetivo do modelo DEA aqui proposto é medir o desempenho da decisão do produtor do sistema de cria. Dessa forma, as DMUs do modelo DEA são os 21 sistemas modais de cria, identificados a partir dos painéis de discussão em 21 municípios de sete estados brasileiros.

\subsection{Variáveis}

Conforme destacam Gomes et al. (2009a), a etapa de seleção das variáveis em DEA é de grande importância, pois dela depende a qualidade dos resultados. Um modelo com grande número de variáveis pode acarretar uma avaliação extremamente benevolente, com várias DMUs 100\% eficientes. Para o caso de avaliação da decisão do produtor vinculado ao sistema de cria, as variáveis do modelo foram escolhidas dentre aquelas de caráter produtivo, já que os índices zootécnicos são menos passíveis de controle por parte do pecuarista.

Os técnicos envolvidos no projeto, ao analisarem esse conjunto de variáveis, identificaram imediatamente aquelas relevantes para o estudo em questão. Como input foi selecionada a quantidade de reprodutores, já que essa variável representa parte significativa dos gastos totais dos pecuaristas que produzem bezerro, sendo diretamente ligada à qualidade dos animais que serão comercializados nesses sistemas. Esta também é a única categoria de animal do rebanho de cria que é adquirida de outros rebanhos, especialmente de fazendas com plantéis de seleção genética.

Como outputs foram escolhidos os produtos do sistema que geram as principais receitas da pecuária de cria. São esses a quantidade de bezerros na propriedade e a quantidade de vacas de descarte. Todos os bezerros produzidos e existentes na propriedade são vendidos e geram renda. As vacas de descarte são vendidas por não mais fazerem parte do sistema produtivo, seja por idade ou por desempenho reprodutivo inferior ao desejado.

As variáveis indicadas por especialistas necessitam ser examinadas pelos analistas para verificar se estão de acordo com as propriedades requeridas pelos modelos DEA. Em particular, deve haver uma relação causal entre cada par input-output (GOMES et al. 2009a, b). É evidente a relação causal entre o output quantidade de bezerros na propriedade e o input quantidade de reprodutores. Já o mesmo não se pode dizer da relação entre o input e o output quantidade de vacas de descarte. Na verdade não há uma relação causal direta entre essas variáveis. Há, no entanto, uma relação custo-benefício. Se o produtor tiver mais reprodutores (que representam um gasto), ele deve arrecadar mais, seja com a venda de bezerros ou de vacas. Então, o par reprodutores-vacas de descarte faz sentido quando se usa DEA para analisar relações custo-benefício e não apenas relações produtivas puras. Essa interpretação de DEA foi introduzida por Womer et al. (2006) e usada por Kuosmanen e Kortelainen (2007), Kuosmanen, Bijsterbosch e Dellink (2009) e Barros et al. (2010). Generalizações desse uso podem ser vistas em Bougnol et al. (2010).

Na Tabela 1 são apresentados os dados. Verifica-se que os rebanhos são de tamanhos diferentes, o que se reflete nas diferentes escalas de produção. Todos os sistemas apresentam a aquisição de touros reprodutores como base para produzir bezerros de melhor qualidade.

\subsection{Modelo}

Quanto à escolha do modelo DEA, neste artigo foi usado o modelo DEA BCC, já que não havia evidências de proporcionalidade entre input e outputs e as escalas de produção são sabidamente diferentes entre os sistemas modais dos diferentes municípios. Uma vez que o modelo CCR em sua forma fracionária apresenta uma função homogênea de grau zero, seria necessária essa proporcionalidade para justificar o seu uso.

Foi escolhida a orientação a inputs, visto que 0 objetivo do modelo é avaliar o desempenho quanto à decisão do pecuarista que se baseia, quando da compra de touros, na maior eficiência da utilização dos reprodutores mantidos na propriedade, como estratégia de diminuir custos.

Neste caso de estudo há diferença de importância entre as variáveis de output. Os bezerros são o principal produto de venda dos sistemas modais de cria. Adicionalmente, quanto maior forem os índices reprodutivos do rebanho, a renda da venda dos bezerros é mais importante que a venda das matrizes 
Tabela 1. Variáveis do modelo: input e outputs.

\begin{tabular}{|c|c|c|c|c|}
\hline \multicolumn{2}{|l|}{ DMUs } & \multirow{2}{*}{ Reprodutores (input) } & \multirow{2}{*}{ Bezerros (output) } & \multirow{2}{*}{ Vacas de descarte (output) } \\
\hline Município & Estado & & & \\
\hline Alvorada & TO & 12 & 147 & 30 \\
\hline Amambaí & MS & 15 & 143 & 40 \\
\hline Aquidauana & MS & 92 & 713 & 214 \\
\hline Bonito & MS & 14 & 166 & 75 \\
\hline Brasilândia & MS & 31 & 290 & 178 \\
\hline Camapuã & MS & 9 & 65 & 33 \\
\hline Carlos Chagas & MG & 19 & 297 & 160 \\
\hline Catalão & GO & 8 & 81 & 42 \\
\hline Corumbá & MS & 69 & 455 & 200 \\
\hline 1tamarajú & BA & 4 & 44 & 18 \\
\hline Lavras do Sul & RS & 5 & 58 & 30 \\
\hline Montes Claros & MG & 5 & 47 & 28 \\
\hline Niquelândia & GO & 4 & 35 & 18 \\
\hline Paraíso do Tocantins & TO & 12 & 123 & 35 \\
\hline Porangatu & GO & 5 & 46 & 23 \\
\hline Ribas do Rio Pardo & MS & 15 & 143 & 70 \\
\hline Rio Verde & GO & 23 & 196 & 82 \\
\hline São Gabriel D’Oeste & MS & 11 & 95 & 40 \\
\hline Tupã & $\mathrm{SP}$ & 5 & 46 & 30 \\
\hline Uberaba & MG & 5 & 66 & 36 \\
\hline Uberlândia & MG & 2 & 20 & 10 \\
\hline
\end{tabular}

de descarte. Essa informação foi acrescentada ao modelo na forma de restrições aos pesos.

As restrições do tipo inputs e outputs virtuais apresentam como desvantagens a alta probabilidade de ocorrerem inviabilidades nos PPLs de DEA (LINS; SILVA; LOVELL, 2007) e a necessidade de mais informação por parte do decisor que pode até ter que se amparar em métodos mais sofisticados para conseguir prover a informação necessária (SOARES DE MELLO et al., 2002). Por esse motivo preferiu-se usar restrições do tipo ARI. É importante destacar que ao se impor restrições aos pesos do tipo ARl, se o interesse for medir a importância atribuída por cada DMU a cada variável, devem-se normalizar os dados. Evita-se, assim, que os valores resultantes dos pesos não sejam influenciados pela escala dos dados.

\section{Resultados}

A Tabela 2 traz os resultados de eficiência, multiplicadores e fatores de escala calculados segundo o modelo BCC, com orientação a inputs e restrição do tipo ARl peso da variável bezerro > peso da variável vaca de descarte.

Dos 21 sistemas modais de cria analisados, quatro foram DEA BCC eficientes DMUs: Aquidauana, Carlos Chagas, Uberaba, Uberlândia. Uberlândia tem o menor valor de input e Aquidauana o maior valor em ambos os outputs. Essas duas DMUs são conhecidas como eficientes por default. A eficiência delas seria sempre unitária, não importando o valor das outras variáveis. São DMUs cuja eficiência pode ser apenas uma distorção matemática e deve haver algum cuidado ao indicá-las como benchmarks. Em particular Uberlândia, que atribuiu valores nulos aos multiplicadores dos outputs, o que indica que só obteve a eficiência devido à sua pequena escala.

Os três sistemas de produção desenvolvido em municípios de MG foram os que apresentaram maior taxa de natalidade, em torno de $80 \%$. Os sistemas modais de Uberaba e Uberlândia podem ser considerados de pequena escala e o de Carlos Chagas pode ser considerado médio. Nesses três municípios os índices reprodutivos das matrizes foram muito bons, o que reflete boas práticas zootécnicas com uso eficiente dos reprodutores. Ou seja, os sistemas apresentaram proporcionalmente maior produção de bezerros para venda, com menor número de touros adquiridos (dentro da escala de cada sistema). Carlos Chagas e Uberaba operaram com retorno decrescente à escala, ou seja, poderiam diminuir o input para produzir a mesma quantidade de bezerros.

Em Aquidauana, o sistema de cria avaliado foi no Pantanal, onde a criação de bovinos é realizada de maneira extensiva, devido às peculiaridades do bioma. 0 número de reses nos rebanhos de cria é grande e, consequentemente, o produtor trabalha de maneira geral com grande escala para desenvolver 
Tabela 2. Resultados do modelo DEA BCC com restrições aos pesos: eficiência, multiplicadores e fatores de escala.

\begin{tabular}{|c|c|c|c|c|c|}
\hline \multirow{2}{*}{ DMU } & \multirow{2}{*}{ Eficiência } & \multicolumn{3}{|c|}{ Multiplicadores } & \multirow{2}{*}{$\mathrm{u}^{*}$} \\
\hline & & REP & BEZ & VDESC & \\
\hline Alvorada & 0,8056 & 7,4174 & 3,6237 & 0 & 0,0580 \\
\hline Amambaí & 0,6640 & 6,2721 & 3,0642 & 0 & 0,0490 \\
\hline Aquidauana & 1,0000 & 1,0000 & 1,3522 & 0 & $-0,3522$ \\
\hline Bonito & 0,7997 & 6,5742 & 3,2118 & 0 & 0,0514 \\
\hline Brasilândia & 0,8451 & 3,0015 & 2,8328 & 2,8328 & $-2,6641$ \\
\hline Camapuã & 0,5207 & 9,9405 & 4,8564 & 0 & 0,0777 \\
\hline Carlos Chagas & 1,0000 & 4,7290 & 2,3103 & 0 & 0,0370 \\
\hline Catalão & 0,7322 & 11,5565 & 5,6458 & 0 & 0,0903 \\
\hline Corumbá & 0,7983 & 1,3356 & 1,2605 & 1,2605 & $-1,1855$ \\
\hline 1tamarajú & 0,8515 & 22,4170 & 10,9517 & 0 & 0,1752 \\
\hline Lavras do Sul & 0,8636 & 18,1478 & 8,8660 & 0 & 0,1418 \\
\hline Montes Claros & 0,7577 & 17,9149 & 3,0512 & 3,0512 & 0,1571 \\
\hline Niquelândia & 0,7704 & 24,2583 & 11,8512 & 0 & 0,1896 \\
\hline Paraíso do Tocantins & 0,7302 & 7,9233 & 3,8709 & 0 & 0,0619 \\
\hline Porangatu & 0,7494 & 18,9378 & 9,2519 & 0 & 0,1480 \\
\hline Ribas do Rio Pardo & 0,6332 & 5,9814 & 2,9222 & 0 & 0,0468 \\
\hline Rio Verde & 0,5641 & 3,9626 & 1,9359 & 0 & 0,0310 \\
\hline São Gabriel D’Oeste & 0,6132 & 8,4048 & 4,1061 & 0 & 0,0657 \\
\hline Tupã & 0,7708 & 17,7376 & 3,0210 & 3,0210 & 0,1555 \\
\hline Uberaba & 1,0000 & 18,7860 & 3,1995 & 3,1995 & 0,1647 \\
\hline Uberlândia & 1,0000 & 46,5221 & 0 & 0 & 1,0000 \\
\hline
\end{tabular}

Linhas em itálico destacam DMUs eficientes.

a atividade (ABREU; McMANUS; SANTOS, 2010). 0 sistema de produção, apesar da maior escala, foi caracterizado pelo uso eficiente dos touros, mas com retorno crescente à escala. Existe a possibilidade de se utilizar mais input (touros), o que possibilitará aumento de produção de bezerros (output).

Note-se que a maioria das DMUs desconsiderou o output VDESC no cálculo da medida de eficiência. Poder-se-ia argumentar que foi devido à restrição imposta. Entretanto, cabe ressaltar que o modelo calculado sem essa restrição apresentou resultado similar: das 21 DMUs, 15 atribuíram peso zero a essa variável. Isso mostra que a maioria dos produtores toma as decisões em relação à aquisição de touros sem considerar a dinâmica da reposição e descarte de matrizes. As compras dos touros são efetuadas mais em função do preço dessa categoria em relação ao custo de produção e ao mercado do bezerro do que com ajuste ao número das vacas do rebanho de cria.

É interessante também destacar que no modelo sem restrições aos pesos, as DMUs Brasilândia, Carlos Chagas, Corumbá e Montes Claros atribuíram peso maior à variável vacas de descarte. Provavelmente porque nos sistemas modais de Brasilândia, Carlos Chagas e Montes Claros as taxas de descarte de vacas são em torno de 18\%, sendo a média de descarte na amostra analisada de $14 \%$. Portanto, são mais dinâmicos no descarte e reposição de fêmeas para compor o rebanho. Em Corumbá, o sistema é muito extensivo (por ser no Pantanal), além de apresentar indices reprodutivos inferiores (taxa de natalidade, taxa de desmama etc.). Consequentemente, o descarte de fêmeas ganha importância na geração de renda em sistemas com essa característica (ABREU; CEZAR; TORRES, 2003).

Os fatores de escala permitem inferir que apenas três sistemas de cria operam em região de retornos decrescentes de escala, enquanto que os demais estão na faixa de retornos crescentes. Na prática, significa que os produtores podem produzir mais com a aquisição de reprodutores, mas provavelmente devido ao custo alto dos animais em relação os baixos preços do mercado de bezerros dos últimos anos (ABREU; CARVALHO; MORAES, 2008) os produtores foram avessos a correr risco de endividamento para a compra de reprodutores.

Quanto aos resultados do modelo do Envelope, conforme mostrado na Tabela 3, as DMU Carlos Chagas e Uberlândia foram os sistemas de cria mais referenciados como benchmark (76\% de contribuições não nulas na formação do alvo das DMUs ineficientes). Esses sistemas produtivos podem servir de referência para os demais quando se avalia o desempenho da decisão do pecuarista em relação a critérios de produção. Entretanto, cabe ressalvar novamente que a DMU Uberlândia foi eficiente por default e que deve haver algum cuidado ao indicá-las como benchmark. 
3. Contribuições das DMUs eficientes na formação dos alvos das ineficientes.

\begin{tabular}{|c|c|c|c|c|}
\hline & Aquidauana & Carlos Chagas & Uberaba & Uberlândia \\
\hline Alvorada & 0 & 0,4586 & 0 & 0,5414 \\
\hline Amambaí & 0 & 0,4441 & 0 & 0,5559 \\
\hline Aquidauana & 1 & 0 & 0 & 0 \\
\hline Bonito & 0 & 0,5272 & 0 & 0,4728 \\
\hline Brasilândia & 0,0889 & 0,9111 & 0 & 0 \\
\hline Camapuã & 0 & 0,1626 & 0 & 0,8374 \\
\hline Carlos Chagas & 0 & 1,0000 & 0 & 0 \\
\hline Catalão & 0 & 0,2204 & 0 & 0,7796 \\
\hline Corumbá & 0,4898 & 0,5102 & 0 & 0 \\
\hline Itamarajú & 0 & 0,0868 & 0 & 0,9132 \\
\hline Lavras do Sul & 0 & 0,1373 & 0 & 0,8627 \\
\hline Montes Claros & 0 & 0 & 0,6554 & 0,3446 \\
\hline Niquelândia & 0 & 0,0540 & 0 & 0,9460 \\
\hline Paraíso do Tocantins & 0 & 0,3720 & 0 & 0,6280 \\
\hline Porangatu & 0 & 0,0952 & 0 & 0,9048 \\
\hline Ribas do Rio Pardo & 0 & 0,4441 & 0 & 0,5559 \\
\hline Rio Verde & 0 & 0,6363 & 0 & 0,3637 \\
\hline São Gabriel D’Oeste & 0 & 0,2709 & 0 & 0,7291 \\
\hline Tupã & 0 & 0 & 0,6919 & 0,3081 \\
\hline Uberaba & 0 & 0 & 1,0000 & 0 \\
\hline Uberlândia & 0 & 0 & 0 & 1,0000 \\
\hline
\end{tabular}

\section{Considerações finais}

Na fase de cria na pecuária de corte, em função de ela possuir maior risco e menor remuneração, os produtores buscam maneiras de ter menores custos de produção, sendo cautelosos em realizar gastos maiores como adquirir reprodutores. Assim, a maioria dos sistemas avaliados trabalha com retornos crescentes à escala e perdem em eficiência. Ou seja, poderiam produzir mais e de forma ajustada à escala se investissem em balancear melhor o número de reprodutores nos rebanhos de cria. Os sistemas modais de produção dos municípios de Carlos Chagas (MG) e Uberlândia (MG) são exemplos desse balanceamento entre investimento em touros e maior produção de bezerros para venda, dentro de escalas compatíveis com a renda dos produtores.

0 descarte de vacas, outra importante fonte de renda do produtor de cria, depende diretamente da dinâmica que o produtor decide dar à reposição de matrizes no rebanho. Em sistemas com alta taxa de descarte, essa decisão deve ser equilibrada com taxas de desmame que garantam a reposição das vacas por novilhas. Sistemas com índices reprodutivos mais eficientes, que permitem alta taxa de descarte, ou sistemas com maior escala têm o descarte de matrizes como fonte de renda até mais importante que a venda de bezerros. Isso sinaliza a possibilidade de melhorar a condição de renda por meio da venda de vacas de descarte gordas, aumentando o valor dos animais na venda e, dessa forma, melhorando a renda.

\section{Referências}

ABREU, U. G. P.; CARVAlHO, T. B.; MORAES, A. S. Análise do Preço do Bezerro Pago no Pantanal da Nhecolândia, no período de 2001 a 2008. Corumbá: Embrapa Pantanal, 2008. 7 p. il. (Embrapa Pantanal, Comunicado Técnico, n. 70).

ABREU, U. G. P.; CARVALHO, T. B.; ZEN, S. Caracterização produtiva dos sistemas de cria de gado de corte no Brasil. In: REUNIÃO ANUAL DA SOCIEDADE BRASILEIRA DE ZOOTECNIA, 47., 2010, Salvador. Anais... Salvador, 2010. CD-ROM.

ABREU, U. G. P.; CEZAR, 1. M.; TORRES, R. A. Análise bioeconômica da introdução de período de monta em sistemas de produção de rebanhos de cria na região do Brasil Central. Revista Brasileira de Zootecnia, v. 3, n. 5, p. 1198-1206, 2003. http://dx.doi.org/10.1590/S151635982003000500021

ABREU, U. G. P. et al. Avaliação sistêmica da introdução de tecnologias na pecuária de gado de corte do Pantanal por meio de modelos de análise envoltória de dados (DEA). Revista Brasileira de Zootecnia, v. 37, n. 11, p. 2069-2076, 2008. http://dx.doi.org/10.1590/ S1516-35982008001100025

ABREU, U. G. P. et al. Heifer retention program in the Pantanal: a study with data envelopment analysis (DEA) and Malmquist index. Revista Brasileira de Zootecnia, v. 41, n. 8, p. 1937-1943, 2012. http://dx.doi. org/10.1590/S1516-35982012000800019

ABREU, U. G. P. et al. Avaliação da introdução de tecnologias no sistema de produção de gado de corte no Pantanal. Análise de eficiência. Revista Brasileira de Zootecnia, v. 35, n. 3, p. 1242-1250, 2006. http://dx.doi. org/10.1590/S1516-35982006000400040

ABREU, U. G. P.; McMANUS, C.; SANTOS, S. A. Cattle ranching, conservation and transhumance in the 
Brazilian Pantantal. Pastoralism - Research, Policy and Practice, v. 1, p. 99-114, 2010.

ADLER, N.; FRIEDMAN L.; SINUANY-STERN, Z. Review of ranking methods in the data envelopment analysis context. European Journal of Operational Research, v. 140, p. 249-265, 2002. http://dx.doi.org/10.1016/ S0377-2217(02)00068-1

ALLEN, R. et al. Weights restrictions and value judgements in data envelopment analysis: evolution, development and future directions. Annals of Operations Research, v. 73, p. 13-34, 1997. http://dx.doi. org/10.1023/A:1018968909638

ANGULO MEZA, L.; LINS, M. P. E. Review of methods for increasing discrimination in data envelopment analysis. Annals of Operations Research, v. 116, p. 225-242, 2002. http://dx.doi.org/10.1023/A:1021340616758

BANKER, R. D.; CHARNES, A.; COOPER, W. W. Some models for estimating technical scale inefficiencies in data envelopment analysis. Management Science, v. 30, n. 9, p. 1078-109, 1984. http://dx.doi.org/10.1287/ mnsc.30.9.1078

BARROS, T. D. et al. Avaliação dos atrasos em transporte aéreo com um modelo DEA. Produção, v. 20, n. 4, p. 601-611, 2010. http://dx.doi.org/10.1590/S010365132010005000047

BOUGNOL, M. L. et al. Enhancing standard performance practices with DEA. Omega, v. 38, n. 1-2, p. 33-45, 2010. http://dx.doi.org/10.1016/j.omega.2009.02.002

CAPORALETTI, L. E.; DULÁ, J. H.; WOMER, N. K. Performance evaluation based on multiple attributes with nonparametric frontiers. Omega, v. 27, n. 6, p. 637-645, 1999. http://dx.doi.org/10.1016/S03050483(99)00022-5

CARDOSO, E. G. A cadeia produtiva da pecuária bovina de corte. Campo Grande: Embrapa-CNPGC, 1994. 17 p. (Embrapa-CNPGC. Documentos, n. 49).

CARVAlHO, T. B.; ZEN, S.; TAVARES, É. C. N. Comparação de custo de produção na atividade de pecuária de engorda nos principais países produtores de carne bovina. In: Reunião da Sociedade Brasileira de Economia, Administração e Sociologia Rural, 47., 2009, Porto Alegre. Anais... Porto Alegre, 2009.

CARVALHO, T. B.; ZEN, S.; FERREIRA, P. C. Caracterização da atividade pecuária de engorda nos principais países produtores de carne bovina. In: Reunião da Sociedade Brasileira de Economia, Administração e Sociologia Rural, 46., 2008, Rio Branco. Anais... Rio Branco, 2008.

CENTRO DE ESTUDOS AVANÇADOS EM ECONOMIA APLICADA - CEPEA. Metodologia do índice de preços dos insumos utilizados na produção pecuária brasileira. Confederação da Agricultura e pecuária do Brasil, 2010. Disponivel em: <http://www.cepea.esalq.usp.br/boi/ metodologiacna.pdf>. Acesso em: 24 mar. 2010.

CHARNES, A.; COOPER, W. W.; RHODES, E. Measuring the efficiency of decision-making units. European Journal of Operational Research, v. 2, p. 429-444, 1978. http:// dx.doi.org/10.1016/0377-2217(78)90138-8

EUCLIDES FILHO, K. A pecuária de corte brasileira no terceiro milênio. In: SIMPÓSIO SOBRE O CERRADO, 8.; INTERNACIONAL SYMPOSIUM ON TROPICAL SAVANNAS, 1., 1996, Brasília. Biodiversidade e produção sustentável de alimentos e fibras nos cerrados. Anais... Planaltina: EMBRAPA-CPAC, 1996. p. 118-120.
EUCLIDES FILHO, K. Produção de bovinos de corte e o trinômio genótipo - ambiente - mercado. Campo Grande: Embrapa Gado de Corte, 2000. 61 p. il. (Embrapa Gado de Corte, Documentos, n. 85).

GOMES, E. G. Uso de modelos DEA em agricultura: Revisão de literatura. Engevista, v.10, p. 27-51, 2008.

GOMES, E. G. et al. Unitary input DEA model to identify beef cattle production systems typologies. Pesquisa Operacional, v. 32, n. 2, p. 389-406, 2012. http://dx.doi. org/10.1590/S0101-74382012005000015

GOMES, E. G. et al. Dependência espacial da eficiência do uso da terra em assentamento rural na Amazônia. Produção, v. 19, n. 2, p. 417-432, 2009a. http://dx.doi. org/10.1590/S0103-65132009000200015

GOMES, E. G. et al. Efficiency and sustainability assessment for a group of farmers in the Brazilian Amazon. Annals of Operations Research, v. 169, n. 1, p. 167-181, 2009b. http://dx.doi.org/10.1007/s10479-008-0390-6

GOMES, E. G.; SOARES DE MELLO, J. C. C. B.; MANGABEIRA, J. A. C. Estudo da sustentabilidade agrícola em um município amazônico com análise envoltória de dados Pesquisa Operacional, v. 29, p. 23-42, 2009. http:// dx.doi.org/10.1590/S0101-74382009000100002

KUOSMANEN, T.; BIJSTERBOSCH, N.; DELLINK, R. Environmental cost-benefit analysis of alternative timing strategies in greenhouse gas abatement: A data envelopment analysis approach. Ecological Economics, v. 68, n. 6, p. 1633-1642, 2009. http://dx.doi. org/10.1016/j.ecolecon.2008.07.012

KUOSMANEN, T.; KORTELAINEN, M. Valuing environmental factors in cost-benefit analysis using data envelopment analysis. Ecological Economics, v. 62, n. 1, p. 56-65, 2007. http://dx.doi.org/10.1016/j.ecolecon.2007.01.004

LAMPERT, V. N. et al. Development and application of a bioeconomic efficiency index for beef cattle production in Rio Grande do Sul, Brazil. Revista Brasileira de Zootecnia, v. 41, n. 3, p. 775-782, 2012. http://dx.doi. org/10.1590/S1516-35982012000300042

LINS, M. P.; ANGULO MEZA, L. Análise Envoltória de Dados e perspectivas de integração no ambiente de Apoio à Decisão. Rio de Janeiro: Editora da COPPE/UFRJ, 2000.

LINS, M. P. E.; SILVA, A. C. M.; LOVELL, C. A. K. Avoiding infeasibility in DEA models with weight restrictions. European Journal of Operational Research, v. 181, p. 956-966, 2007. http://dx.doi.org/10.1016/j. ejor.2006.05.041

PLAXICO, J. S.; TWEETEN, L. G. Representative farms for policy and projection research. Journal of Farm Economics, v. 45, p. 1458-1465, 1963. http://dx.doi. $\operatorname{org} / 10.2307 / 1236844$

SOARES DE MELLO, J. C. C. B.; ANGULO MEZA, L.; BRANCO DA SILVA, B. P. A ranking for the Olympic Games with unitary input DEA models. IMA Journal of Management Mathematics, v. 20, n. 2, p. 201-211, 2009. http://dx.doi. org/10.1093/imaman/dpn025

SOARES DE MELLO, J. C. C. B. et al. Cross evaluation using weight restriction in unitary input DEA models: Theoretical aspects and application to Olympic Games ranking. WSEAS Transactions on Systems, v. 7, n. 1 , p. 31-39, 2008a.

SOARES DE MELLO, J. C. C. B. et al. DEA Advanced Models for Geometric Evaluation of used lathes. WSEAS Transactions on Systems, v. 7, n. 5, p. 510-520, 2008 b. 
SOARES DE MELLO, J. C. C. B. et al. Evaluating the performance of Calculus classes using operational research tools. European Journal of Engineering Education, v. 27, n. 2, p. 209-218, 2002. http://dx.doi. org/10.1080/03043790210129577

THANASSOULIS, E.; PORTELA, M. C. S.; ALLEN, R. Incorporating value judgments in DEA. In: COOPER, W. W.; SEIFORD, L. M.; ZHU, J. (Eds.). Handbook on data envelopment analysis. Boston: Kluwer Academic Publishers, 2004. p. 99-138.

THOMPSON, R. G. et al. The role of multiplier bounds in efficiency analysis with application to Kansas farming.
Journal of Econometrics, v. 46, p. 93-108, 1990. http:// dx.doi.org/10.1016/0304-4076(90)90049-Y

WOMER, N. K. et al. Benefit-cost analysis using data envelopment analysis. Annals of Operations Research, v. 145, n. 1, p. 229-250, 2006. http://dx.doi.org/10.1007/ s10479-006-0036-5

\section{Agradecimentos}

À Embrapa (Macroprograma 1), à Faperj e ao $\mathrm{CNPq}$, pelo apoio financeiro.

\section{Performance evaluation of livestock production systems in Brazil}

\section{Abstract}

In this article, the performance of extensive livestock modal production systems was analyzed comparatively during its rearing phase in 21 Brazilian municipalities. This phase constitutes the activity that sustains the entire livestock production chain. Data envelopment analysis (DEA) models were used with variable returns to scale. The objective was to measure the performance of the producer's decision regarding the composition of the production system. The number of bull breeders, number of calves and number of cull cows were the variables used. Four of the 21 systems evaluated were DEA efficient and most of them were operating under increasing returns to scale.

\section{Keywords}

Efficiency. DEA. Livestock. Modal systems. 